REVIEW

\title{
Severe acute respiratory syndrome (SARS): epidemiology and clinical features
}

\author{
D S C Hui, M C H Chan, A K Wu, P C Ng
}

Postgrad Med J 2004;80:373-381. doi: 10.1136/pgmj.2004.020263

Severe acute respiratory syndrome (SARS) is a newly emerged infectious disease with a significant morbidity and mortality. The major clinical features include persistent fever, chills/rigor, myalgia, malaise, dry cough, headache, and dyspnoea. Older subjects may present without the typical febrile response. Common laboratory features include lymphopenia, thrombocytopenia, raised alanine transaminases, lactate dehydrogenase, and creatine kinase. The constellation of compatible clinical and laboratory findings, together with certain characteristic radiological features and lack of clinical response to broad spectrum antibiotics, should arouse suspicion of SARS. Measurement of serum RNA by real time reverse transcriptase-polymerase chain reaction technique has a detection rate of $75 \%-80 \%$ in the first week of the illness.

See end of article for authors' affiliations

Correspondence to:

Dr D S C Hui, Department of Medicine and Therapeutics, Chinese University of Hong Kong, Prince of Wales Hospital, Shatin, New Territories, Hong Kong; dschui@cuhk. edu.hk

Submitted 9 February 2004 Accepted 26 February 2004
S evere acute respiratory syndrome (SARS) is a newly emerged infectious disease in the 2 lst century that has posed an enormous threat to international health. In February and March 2003 there were major outbreaks of SARS in Hong Kong, ${ }^{1}$ Singapore, ${ }^{2}$ Vietnam, Taiwan, Canada, ${ }^{3}$ and other countries. As of 31 July 2003, 8098 probable cases were reported in 29 countries and regions with a death toll of 774 $(9.6 \%) .{ }^{4}$ A novel coronavirus has been identified as the pathogen responsible for SARS. ${ }^{5-8}$ Several laboratories have completed sequencing the genome of the coronavirus that has led to the global epidemic of SARS, and noted that the SARS coronavirus (SARS CoV) is not closely related to any of the previously characterised coronaviruses. $^{9-11}$

\section{EPIDEMIOLOGY}

\section{China}

The early cases of SARS appeared to have originated in southern China. In November 2002, there was an unusual epidemic of severe pneumonia of unknown aetiology in Foshan, Guangdong Province in southern China, with a high rate of transmission to healthcare workers. ${ }^{12}$ A retrospective analysis of 55 patients admitted to a chest hospital with atypical pneumonia in Guangzhou between 24 January and 18 February 2003 showed positive SARS CoV in the nasopharyngeal aspirates, whereas 48 (87\%) patients had positive antibodies to SARS CoV in their convalescent sera. Genetic analysis showed that the SARS CoV isolates from Guangzhou shared the same origin with those in other countries, with a phylogenetic pathway that matched the spread of SARS to other parts of the world. ${ }^{13}$ By April 2003, the epidemic reached its peak in Beijing and several major cities. By the end of the SARS epidemic, there were 5327 probable cases within mainland China with a mortality rate of $7 \%$.

\section{Hong Kong}

A 64 year old nephrologist from southern China, who visited Hong Kong on 21 February and died on 4 March 2003 of severe pneumonia, is believed to have been the source of infection causing subsequent outbreaks of SARS in Hong Kong, ${ }^{1}{ }^{14}$ Vietnam, Singapore, ${ }^{2}$ Canada, ${ }^{3}$ and elsewhere. At least 16 hotel guests and visitors had been infected by the Guangdong physician while they were either visiting friends or staying on the same floor of the hotel in Kowloon. The deceased nephrologist was subsequently proven to have positive reverse transcriptase-polymerase chain reaction (RT-PCR) on retrospective virological analysis of both the nasopharyngeal aspirate taken before death and the postmortem lung, in addition to a fourfold rise in antibody titre against SARS CoV. ${ }^{15}$ As a result of the relatively long incubation period of up to 10-14 days in some cases, SARS spread rapidly and globally by international travellers to their home cities without any symptoms before their arrival.

SARS appears to spread by close person-toperson contact via droplet transmission or fomite. ${ }^{16}$ The high infectivity of this viral illness is highlighted by the fact that 138 patients (mostly healthcare workers) were hospitalised with SARS within two weeks as a result of exposure to one single patient on a general medical ward in Hong Kong. ${ }^{1}$ The index case of this major hospital outbreak was a 26 year old man, previously healthy, who had visited a friend on the same floor of hotel in Kowloon as the nephrologist on 21 February 2003. He developed flu-like illness three days later and was admitted to the Prince of Wales Hospital on 4 March 2003 with right upper lobe pneumonia. The use of a jet nebuliser for administering bronchodilator for its mucociliary clearance effects to this patient, who had presented clinically with community acquired pneumonia, could increase the viral droplet load around the patient and, together with overcrowding on the hospital

Abbreviations: SARS, severe acute respiratory syndrome; RT-PCR, reverse transcriptase-polymerase chain reaction; SARS CoV, SARS coronavirus; WHO, World Health Organisation 
ward and poor ventilation, had contributed to this major hospital outbreak. ${ }^{1}$ Interestingly, this index case recovered completely within two weeks after admission without receiving any specific treatment other than conventional antibiotics for community acquired pneumonia. ${ }^{17}$ Based on the information related to the major outbreak at the Prince of Wales Hospital, the World Health Organisation (WHO) placed the international community on high alert on 12 March 2003 about cases of acute respiratory syndrome with unknown aetiology in Vietnam, Hong Kong, and Guangdong that appeared to put healthcare workers at high risk. ${ }^{18}$

The main community outbreak of SARS in Hong Kong occurred in late March at the Amoy Gardens, a private residential estate, where 329 residents were infected. The index case for this outbreak appeared to be a male patient, who worked and lived in Shenzhen in southern China, with end stage renal failure due to systemic lupus erythematosus. ${ }^{19}$ He required haemodialysis twice a week at the Prince of Wales Hospital. He was admitted to the hospital on 15 March 2003 with fever, chills, rigor, and right lower lobe consolidation. Rapid antigen test of his nasopharyngeal aspirate revealed influenza A virus as an alternative diagnosis. He initially responded well to treatment with oseltamivir, cefotaxime, and levofloxacin with defervescence within 48 hours and almost complete clearing of right lower lobe consolidation four days after admission. He was then discharged from the Prince of Wales Hospital with a diagnosis of influenza, with possibly superimposed bacterial pneumonia. He stayed with his brother overnight at the Amoy Gardens, where the major outbreak of SARS occurred subsequently. Three days after discharge, he was readmitted to hospital with bilateral pneumonia with rapid progression to severe respiratory failure. In retrospect, he had suffered from both influenza A and SARS with an unusual phase 1 disease with almost complete resolution of right lower lobe pneumonia before progression to phase 2 disease. ${ }^{19}$ The genomic sequence of the SARS CoV isolated from this patient and the four identical community cases had only three nucleotide differences, supporting the likelihood of this patient being the index of the Amoy Gardens outbreak, ${ }^{20}$ although it would be difficult to explain the major outbreak of SARS in different residential units in different blocks and different directions at the Amoy Gardens based on one single source. There are several hypotheses for this major outbreak including passive carriage of virus by pests, drying up of a $U$ shaped floor drain, and faecal-oral viral loading through contaminated surfaces as a result of the chimney effects created by the use of exhaust fans in the presence of blockage of the sewage system. ${ }^{21}$ The last case of SARS occurred on 2 June 2003 and Hong Kong was declared SARS-free on 23 June 2003.

\section{Vietnam}

SARS was brought to Hanoi by a Chinese-American businessman who had stayed in a room opposite to that of the nephrologist on the same floor of the hotel. SARS was first identified in Vietnam on 28 February 2003 by Dr Carlo Urbani, a WHO epidemiologist, who died of the disease later in Thailand..$^{22}$ The businessman was transferred to the Princess Margaret Hospital, Hong Kong for further management on 6 March 2003 but died on 13 March 2003 despite intensive care support. There were 63 cases of SARS in Hanoi and it was removed from the list of areas with local transmission on 28 April 2003.

\section{Singapore}

Three guests from Singapore were infected with SARS while staying on the ninth floor of the hotel in Kowloon on 20-21 February 2003. The outbreak in Singapore was characterised by nosocomial transmission involving healthcare workers, and then spread to the community from a SARS patient to two taxi drivers and the patient's co-workers in a wholesale market. ${ }^{23}$ There were 97 (41\%) healthcare workers infected out of 238 probable SARS cases. After prompt and decisive actions by the Singaporean health authorities in implementing contact tracing, isolation, and quarantine measures, the spread of the disease was limited, with the last case being reported on 5 May 2003.

\section{Taiwan}

The first case of SARS occurred in a businessman who had travelled to Guangdong on 5 February 2003, and returned to Taipei via Hong Kong on 21 February 2003. He developed febrile illness on 25 February but was not hospitalised until 8 March 2003. The wife and son of the patient were infected with SARS, and both required invasive mechanical ventilation. For the first six weeks of the SARS outbreak, recognised spread was limited to one healthcare worker and three household contacts. ${ }^{24}$ There was, however, a late but rapid outbreak of SARS in Taiwan from mid-April 2003, and appeared to be related to the visit of a resident of Amoy Gardens to Taiwan on 26 March 2003. Subsequent molecular data analysis has demonstrated that the same strain of the SARS CoV was involved in the Amoy Gardens outbreak and the late outbreak in Taiwan. ${ }^{25}$ Taiwan was the last to be removed on 5 July 2003 from the list of areas with recent local transmission. ${ }^{26}$

\section{Canada}

The index case of SARS in Canada was an elderly woman who returned to Toronto on 23 February 2003 after a visit to Hong Kong. She was exposed to SARS during her stay on the ninth floor of the hotel in Kowloon. She became ill after returning to Toronto and infected her family members. One of her family members was admitted to a community hospital in Toronto and this resulted in a large nosocomial outbreak. ${ }^{27}$ Transmission to other persons resulted subsequently in an outbreak among 257 persons in several hospitals. On 14 May 2003, the WHO removed Toronto from the list of areas with recent local SARS transmission. Unfortunately, after premature relaxation of strict infection control measures such as monitoring of fever and respiratory symptoms in hospitalised patients and visitors, there was a second wave of SARS cases among patients, visitors, and healthcare workers that occurred at a Toronto hospital approximately four weeks after SARS transmission was thought to have been interrupted..$^{28}$ Toronto was finally declared free from local transmission on 2 July $2003 .^{26}$

The statistics of SARS in different parts of the world are shown in table $1 .{ }^{29}$

On 5 July 2003, the WHO announced that the last known chain of human-to-human transmission of the SARS CoV had been broken in Taiwan, and this brought an end to the initial outbreak of SARS that had begun in mid-November 2002 in southern China and spread internationally in late February 2003. It is likely that SARS CoV originated from a wild animal reservoir in mainland China because masked palm civets and the raccoon dog had a CoV almost identical to that in SARS patients and there was a much higher seroprevalence of SARS CoV among wild animal handlers than controls in Guangdong. ${ }^{30}$

\section{CLINICAL FEATURES}

The incubation period of SARS is generally between $2-$ 10 days though it has been estimated as 6.4 days (95\% confidence interval 5.2 to 7.7 ) with a mathematical model. The mean time from onset of clinical symptoms to hospital admission varied between three to five days. ${ }^{31}$ The major clinical features on presentation include persistent fever, chills/rigor, myalgia, malaise, dry cough, headache, and 
Table 1 Summary of probable SARS cases with onset of illness from 1 November 2002 to 31 July $2003^{29}$

\begin{tabular}{lrccll}
\hline Areas & Cumulative No & No of deaths & $\begin{array}{l}\text { Case fatality } \\
\text { ratio (\%) }\end{array}$ & $\begin{array}{l}\text { Age (median } \\
\text { range) years }\end{array}$ & $\begin{array}{l}\text { No (\%) of } \\
\text { healthcare } \\
\text { workers }\end{array}$ \\
\hline Australia & 6 & 0 & 0 & $15(1-45)$ & $1(16)$ \\
Canada & 251 & 43 & 17 & $49(1-98)$ & $109(43)$ \\
China & 5327 & 349 & 7 & Pending & $1002(19)$ \\
Hong Kong & 1755 & 299 & 17 & $40(0-100)$ & $386(22)$ \\
Taiwan & 346 & 37 & 11 & $42(0-93)$ & $68(20)$ \\
Malaysia & 5 & 2 & 40 & $30(26-84)$ & 0 \\
Philippines & 14 & 2 & 14 & $41(29-73)$ & $4(29)$ \\
Singapore & 238 & 33 & 14 & $35(1-90)$ & $97(41)$ \\
Thailand & 9 & 2 & 22 & $42(2-79)$ & $1(11)$ \\
UK & 4 & 0 & 0 & $59(28-74)$ & 0 \\
USA & 29 & 0 & 0 & $33(0-83)$ & 0 \\
Vietnam & 63 & 5 & 8 & $43(20-76)$ & $36(57)$ \\
Global & 8098 & 774 & 9.6 & N/A & $1007(21)$ \\
\hline
\end{tabular}

dyspnoea. Less common symptoms include sputum production, sore throat, rhinorrhoea, nausea and vomiting, and diarrhoea. ${ }^{1-3}$ Nevertheless, these clinical symptoms are rather non-specific and may mimic influenza or atypical pneumonia of other causes such as mycoplasma, chlamydia, and legionella.

Watery diarrhoea, together with recurrence of fever, was reported in $73 \%$ of patients one week into the clinical course in the Amoy Gardens outbreak linked to a faulty sewage system, presumably due to involvement of the gastrointestinal tract via the faecal-oral route. ${ }^{32}$ The diarrhoea was described as watery in large volume but contained no blood or mucus. The maximum frequency of diarrhoea was $6 \pm 4$ times daily and the duration lasted for $3.9 \pm 2.3$ days. Intestinal biopsy specimens taken by colonoscopy or at necropsy revealed minimal architectural disruption but there was evidence of active viral replication within both the small and large intestines. SARS CoV was isolated by culture from these specimens whereas SARS CoV RNA was detected for up to 10 weeks after symptom onset. ${ }^{33}$ The frequency of clinical features on presentation from several case series is summarised in table 2.

SARS CoV has been detected in the cerebrospinal fluid and serum samples of two patients who developed status epilepticus. ${ }^{3435}$ The data suggest that a severe acute neurological syndrome might occasionally accompany SARS.

Older subjects may present with decrease in general wellbeing, poor feeding, fall/fracture, ${ }^{36}$ and in some cases,

\begin{tabular}{ll}
\hline $\begin{array}{l}\text { Table } 2 \text { Clinical features of SARS on } \\
\text { presentation } 1-314\end{array}$ \\
\hline Symptom & $\begin{array}{l}\text { \% Of patients with } \\
\text { symptom }\end{array}$ \\
\hline Persistent fever $>38^{\circ} \mathrm{C}$ & $99-100$ \\
Non-productive cough & $57-75$ \\
Myalgia & $45-61$ \\
Chills/rigor & $15-73$ \\
Headache & $20-56$ \\
Dyspnoea & $40-42$ \\
Malaise & $31-45$ \\
Nausea and vomiting & $20-35$ \\
Diarrhoea & $20-25$ \\
Sore throat & $13-25$ \\
Dizziness & $4.2-43$ \\
Sputum production & $4.9-29$ \\
Coryza & $2.1-23$ \\
Arthralgia & 10.4 \\
\hline
\end{tabular}

delirium, without the typical febrile response (temperature $\left.>38^{\circ} \mathrm{C}\right) .^{36}{ }^{37}$

\section{Laboratory findings}

Lymphopenia (destruction of both CD4 and CD8 lymphocytes), features of low grade disseminated intravascular coagulation (thrombocytopenia, prolonged activated partial thromboplastin time, raised D-dimer), raised lactate dehydrogenase, alanine transaminases, and creatine kinase are common laboratory features of SARS. ${ }^{1-3}{ }^{14} 32$ Absolute lymphopenia occurs in $98 \%$ of cases of SARS during the clinical course of the disease. The CD4 and CD8 T lymphocyte counts fall early in the course of SARS, whereas low counts of CD4 and CD8 at presentation are associated with adverse clinical outcome. $^{38}$

There was also a correlation between the decrease in left ventricular ejection fraction with adverse prognostic factors such as lactate dehydrogenase and creatine kinase levels. ${ }^{39}$ Although the pathogenic mechanism(s) for the cardiac disturbances is unknown, it is possible that that largely reversible subclinical diastolic impairment occurs in acute SARS. ${ }^{39}$

Mildly raised aminotransferase levels are reported in 23\%$50 \%$ of SARS patients, although the clinicopathological significance is largely unknown. ${ }^{14}$ Longitudinal evaluation of liver function was reported on 54 adult SARS patients. Liver dysfunction was found in $29.6 \%$ and $75.9 \%$ of patients on presentation (before ribavirin treatment) and during treatment for SARS with ribavirin and corticosteroid. ${ }^{40}$ Time to maximal radiographic damage, evaluated in the form of an overall aggregate score, correlated with time to peak alanine transaminases thus suggesting a common pathogenic mechanism(s), namely immune mediation for disruption to the lung and liver. ${ }^{41}$

\section{Radiological findings}

The radiographic appearances of SARS share common features with pneumonia of other causes. About 20\%-25\% of patients with SARS may have normal chest radiographs on presentation, ${ }^{13}{ }^{14} 42$ whereas high resolution computed tomography of the thorax is useful in detecting parenchymal opacities early. ${ }^{43}$ In our study, the opacities occupy a peripheral or mixed peripheral and axial location in $88 \%$ of patients. ${ }^{42}$ The predominant involvement of lung periphery and the lower zone, in addition to the absence of cavitation, hilar lymphadenopathy or pleural effusion, are the more distinctive radiographic features of SARS. ${ }^{142}$ Radiographic progression from unilateral focal air-space opacity to either multifocal or bilateral involvement during the second phase 
of the disease, followed by radiographic improvement with treatment, is commonly observed (fig 1). ${ }^{1}{ }^{42}$ In a case series, $12 \%$ of patients developed spontaneous pneumomediastinum and $20 \%$ of patients developed evidence of acute respiratory distress syndrome over a period of three weeks. ${ }^{32}$ In general, the incidence of barotrauma in intensive care admissions
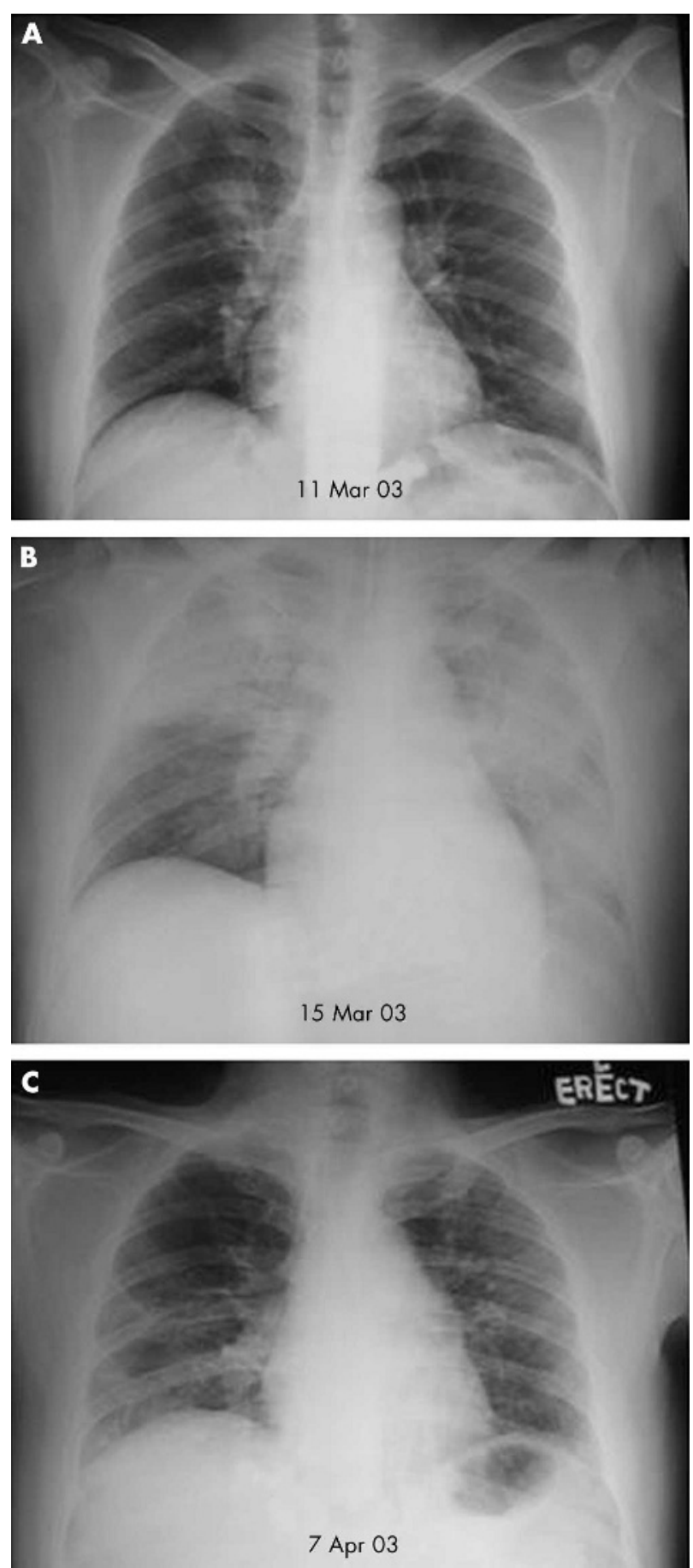

Figure 1 Serial chest radiographs of a 33 year old male healthcare worker who developed fever, myalgia and dry cough on 8 March 2003. (A) The early film (11 March 2003) showed an area of consolidation involving the right upper zone. (B) His condition deteriorated on 15 March 2003 (phase 2 disease) when he required invasive mechanical ventilation for severe respiratory failure. (C) His chest radiograph on 7 April 2003 showed postinflammatory changes in both lung fields. He finally made a full recovery. seems higher than expected despite low volume and low pressure mechanical ventilation.

High resolution computed tomography of the thorax is useful in detecting lung opacities in cases with unremarkable chest radiographs. Common findings include ground glass opacification, sometimes with consolidation, and interlobular septal and intralobular interstitial thickening, with predominantly a peripheral and lower lobe involvement (fig 2). The characteristic peripheral alveolar opacities bear close resemblance to those found in bronchiolitis obliterans organising pneumonia. ${ }^{13}$

\section{CLINICAL COURSE}

The clinical course of SARS appears to follow a typical pattern $^{32}$ : phase 1 (viral replication) is associated with increasing viral load and clinically characterised by fever, myalgia, and other systemic symptoms that generally improve after a few days. Phase 2 (immunopathological damage) is characterised by recurrence of fever, oxygen desaturation, and radiological progression of pneumonia with falls in viral load. The majority of patients will improve with a combination of ribavirin and intravenous pulse steroid therapy but $20 \%-36 \%$ may require intensive care unit admission, and $13 \%-26 \%$ may progress into acute respiratory distress syndrome necessitating invasive ventilatory support. ${ }^{1324445}$ Based on the study by Peiris et al, ${ }^{32}$ the timing of the IgG seroconversion, which seems to start on day 10, seems to correlate with falls in viral load, which occurs from between day 10 and 15, despite the use of pulse methylprednisolone. Peiris et al have shown very clearly a progressive decrease in rates of viral shedding from nasophargynx, stool, and urine from day 10 to day 21 after symptom onset in the 20 patients who had serial measurements with RT-PCR. Thus clinical worsening during phase 2 cannot be explained by uncontrolled viral replication and is most likely the result of immune mediated lung injury due to an over-exuberant host response. ${ }^{32}$

\section{PAEDIATRIC SARS PATIENTS}

Most patients in the paediatric age group have a definitive contact history with adult SARS patients, usually an immediate family member. ${ }^{46}$ Again, fever is the most commonly observed symptom. Other clinical manifestations such as lethargy, headache, dizziness, rhinorrheoa, myalgia, chills and rigors, have also been reported. ${ }^{46-48}$ In contrast, febrile convulsion, sore throat, abdominal signs and symptoms are less frequently encountered. Respiratory features also tend to be vague and non-specific. Dry cough is the most frequent respiratory symptom, but tachypnoea, dyspnoea,

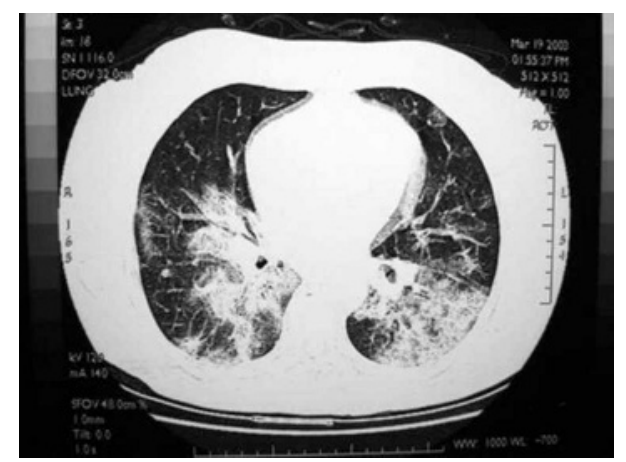

Figure 2 High resolution chest tomography of a patient showing bilateral ground glass opacification involving predominately both lower lobes on day 8 of illness. 
and hypoxaemia are only found in the more severe cases. The most consistent laboratory findings are lymphopenia and raised lactate dehydrogenase levels. Other abnormal haematological and biochemical indices include deranged coagulapathy, thrombocytopenia, raised serum D-dimer, creatine kinase, and transaminase concentrations. The spectrum of radiological abnormalities is also very similar to those of adult SARS patients. Air-space consolidation, predominantly in the lower lobes, is the most frequent finding. ${ }^{49}$

It is now known that the clinical course and prognosis could be very different between paediatric and adult SARS patients. ${ }^{46-48}$ In general, young children $(<12$ years of age) often run a more benign and shorter clinical course, and present with runny rose and cough indistinguishable from the common cold due to respiratory syncytial and other viruses. Conversely, teenage patients tend to have a more protracted and severe course and often present with severe constitutional features, including headache, myalgia, chills and rigors and lower respiratory tract signs, resembling those of adult SARS patients. ${ }^{146}$ However, their clinical manifestations follow a "biphasic" rather than a "triphasic" pattern, as paediatric patients seldom progress to the acute respiratory distress syndrome phase of the disease. There are no fatalities in young children and teenage patients. ${ }^{46-48} 51$ In addition, none of the preterm or term infants born to pregnant women with SARS have been found to be clinically infected or shedding the virus after birth. ${ }^{50}$ All of them follow a clinical course typical of infants with similar gestations..$^{50}$

\section{DIAGNOSTIC CRITERIA OF SARS}

Both the WHO and the Centers for Disease Control and Prevention issued updated case definitions for SARS during the outbreak. A suspected case was defined by the WHO (revised l May 2003) $)^{52}$ as a person presenting after 1 November 2002 with:

- Fever $>38^{\circ} \mathrm{C}$, plus

- Cough or difficulty breathing, plus

- Either close contact with a person who is a suspect or probable case of SARS and/or history of travel or residence in an area with recent local transmission of SARS within 10 days of symptom onset.

Patients with an unexplained fatal acute respiratory illness who fit the above epidemiological criteria but on whom no autopsy has been performed are also classified as suspected cases.

A probable case is defined as:

- A suspected case with radiographic findings of pneumonia or acute respiratory distress syndrome, or

- A suspected case positive for SAR CoV in one or more laboratory assays, or

- A suspected case with necropsy evidence of acute respiratory distress syndrome with unknown cause.

The WHO definitions have been established to assist in the definition of hospital cases. The reason for retaining the clinical and epidemiological basis for the case definitions is that there is as yet no validated, widely and consistently available rapid test for SARS CoV infection. The WHO definitions have been evaluated in the context of screening patients before admission to hospital. ${ }^{53}$ In the early stages of SARS, the main discriminating features are fever, chills, malaise, myalgia, rigors rather than cough and breathing difficulty. Documented fever $\left(>38^{\circ} \mathrm{C}\right)$ may not occur in the early stages in some cases and radiological evidence of pneumonic changes often precedes fever. The WHO case definitions for suspected SARS have a low sensitivity of $26 \%$ and a negative
Box 1: WHO case definitions of SARS in the postoutbreak period 54

Clinical case definition of SARS:

A person with a history of:

- Fever $\geqslant 38^{\circ} \mathrm{C}$, and

- One or more symptoms of lower respiratory tract illness (cough, difficulty breathing, shortness of breath), and

- Radiographic evidence of lung infiltrates consistent with pneumonia or respiratory distress syndrome or necropsy findings consistent with the pathology of pneumonia or respiratory distress syndrome without an identifiable cause, and

- No alternative diagnosis can fully explain the illness.

Laboratory case definition of SARS:

A person with symptoms and signs that are clinically suggestive of SARS and with positive laboratory findings for SARS CoV based on one or more of the following diagnostic criteria:

(A) PCR positive for SARS CoV using a validated method from:

- At least two different clinical specimens (for example, nasopharyngeal aspirate or stool), or

- The same clinical specimen collected on two or more occasions during the course of the illness (for example, sequential nasopharyngeal aspirates), or

- Two different assays or repeat PCR using a new RNA extract from the original clinical sample on each occasion of testing.

(B) Seroconversion by ELISA or immunofluorescence assay:

- Negative antibody test on acute serum followed by positive antibody test on convalescent phase serum tested in parallel, or

- Fourfold or greater rise in antibody titre between acute and convalescent phase sera tested in parallel.

(C) Virus isolation:

- Isolation in cell culture of SARS CoV from any specimen and PCR confirmation using a validated method.

predictive value of $85 \%$ for detecting SARS in patients who have not been admitted to hospital..$^{53}$ The WHO has revised the case definitions in the post-outbreak period with inclusion of radiographic and laboratory findings for public health purposes (box 1)..$^{54}$

The Centers for Disease Control and Prevention case definitions of SARS are based on clinical, epidemiological, and laboratory criteria (box 2).55

The case definitions and exclusion criteria have been revised to allow exclusion of cases with a convalescent phase serum sample, collected $>28$ days after symptom onset, that is negative for antibody to SARS $\mathrm{CoV} .{ }^{55}$ It is important to remember the lesson from the Amoy Gardens index case that an alternative diagnosis, which could fully explain the illness at the time, did not rule out SARS. ${ }^{19}$ 
Box 2: Centers for Disease Control and

\section{Prevention updated interim case definition for} SARS ${ }^{55}$ :

\section{Clinical criteria:}

- Early illness

- Presence of two or more of the following features: fever (might be subjective), chills, rigors, myalgia, headache, diarrhoea, sore throat, rhinorrhoea.

- Mild to moderate respiratory illness

- Temperature $>100.4^{\circ} \mathrm{F}$ or $38^{\circ} \mathrm{C}$ ) and at least one lower respiratory illness (for example, cough, dyspnoea, difficulty breathing).

- Severe respiratory illness

- Meets clinical criteria of mild to moderate respiratory illness, and

- One or more of the following findings: radiographic evidence of pneumonia, or acute respiratory distress syndrome, or necropsy findings consistent with pneumonia, or acute respiratory distress syndrome without an identifiable cause.

Epidemiological criteria:

- Possible exposure to SARS CoV

At least one of the following exposures in the 10 days before onset of symptoms:

- Travel to a foreign or domestic location with documented or suspected recent transmission of SARS, or

- Close contact with a person with mild-to-moderate or severe respiratory illness and with history of travel in the 10 days before onset of symptoms to a foreign or domestic location with documented or suspected recent transmission of SARS CoV.

\section{- Likely exposure to SARS CoV}

One of the following exposures in the 10 days before onset of symptoms:

- Close contact with a confirmed case of SARS CoV disease, or

- Close contact with a person with mild-to-moderate or severe respiratory illness for whom a chain of transmission can be linked to a confirmed case of SARS CoV disease in the 10 days before onset of symptoms.

Laboratory criteria:

- Detection of serum antibody to SARS CoV by a test validated by the Centers for Disease Control and Prevention (CDC) (for example, enzyme immunoassay), or

- Isolation in cell culture of SARS CoV from a clinical specimen, or

- Detection SARS CoV RNA by RT-PCR test validated by CDC and with subsequent confirmation in a reference laboratory (for example, CDC).

\section{Exclusion criteria:}

A person may be excluded as a SARS report under investigation if any of the following applies:

- An alternative diagnosis can fully explain the illness.

- Absence of antibody to SARS CoV in a serum specimen obtained $>28$ days after symptom onset.

- The case was reported on the basis of contact with a person who was excluded subsequently as a case of SARS CoV disease; then the reported case is also excluded provided other epidemiologic or laboratory criteria are absent.

\section{SARS disease classification:}

- Probable case of SARS CoV disease: in a person who meets the clinical criteria for severe respiratory illness and the epidemiological criteria for likely exposure to SARS CoV.

- Confirmed case of SARS CoV disease: in a person who has a clinically compatible illness (that is, early, mildto-moderate, or severe) that is laboratory confirmed.

\section{LABORATORY DIAGNOSIS}

The detection rates for SARS CoV using conventional RT-PCR are generally low in the first week of illness. The positivity rates on urine, nasophargyngeal aspirate, and stool specimen have been reported to be $42 \%, 68 \%$, and $97 \%$ respectively on day 14 of illness whereas serology for confirmation may take 28 days to reach a detection rate above $90 \%{ }^{32}$ By optimising RNA extraction methods and applying quantitative real time RT-PCR techniques, the sensitivity of nasopharyngeal aspirate specimens for early diagnosis of SARS can be enhanced to $80 \%$ for the first three days. ${ }^{56}$ Quantitative measurement of blood SARS CoV RNA with real time RT-PCR technique has been developed with a detection rate of $80 \%$ as early as day 1 of hospital admission but the detection rates drop to $75 \%$ and $42 \%$ on day 7 and day 14 respectively (table 3$)^{57-59}$

\section{TREATMENT}

Because of limited understanding of the pathogenesis and clinical course of this newly emerged disease, treatment of SARS was inevitably empirical during the outbreak in 2003. A retrospective multicentre study has shown that the addition of lopinavir/ritonavir as an initial treatment for SARS was associated with a reduction in the overall death rate $(2.3 \%)$ and intubation rate $(0 \%)$, when compared with a matched cohort who received standard treatment $(15.6 \%$ and $11 \%$ respectively, $\mathrm{p}<0.05$ ), and a lower rate of use of methylprednisolone at a mean dose. ${ }^{60}$ Another study has shown in

Table 3 Diagnostic tests for SARS CoV 32 50-59

\begin{tabular}{|c|c|}
\hline & Detection rate \\
\hline \multicolumn{2}{|l|}{ RT-PCR } \\
\hline Nasophargyneal aspirate & $\begin{array}{l}32 \% \text { day } 3,68 \% \text { day } 14 \\
\text { (conventional RT-PCR) } \\
80 \% \text { with real time quantitative } \\
\text { RT-PCR assay during first } 3 \text { days }\end{array}$ \\
\hline Stool & $97 \%$ day 14 \\
\hline Urine & $42 \%$ day 15 \\
\hline $\begin{array}{l}\text { Real time quantitative serum } \\
\text { SARS CoV RNA }\end{array}$ & $80 \%$ day $1,75 \%$ day $7,45 \%$ day 14 \\
\hline \multicolumn{2}{|l|}{ Serology } \\
\hline $\begin{array}{l}\lg G \text { seroconversion to SARS } \\
\mathrm{CoV}\end{array}$ & $\begin{array}{l}15 \% \text { day } 15,60 \% \text { day } 21,>90 \% \\
\text { day } 28\end{array}$ \\
\hline
\end{tabular}




\section{Key references}

- Lee N, Hui DS, Wu A, et al. A major outbreak of severe acute respiratory syndrome in Hong Kong. N Engl J Med 2003:348:1986-94.

- Kuiken T, Fouchier RA, Schutten M, et al. Newly discovered coronavirus as the primary cause of severe acute respiratory syndrome. Lancet 2003;362:26370.

- Rota PA, Oberste MS, Monroe SS, et al. Characterization of a novel coronavirus associated with severe acute respiratory syndrome. Science 2003;300: 1394-9.

- Guan Y, Zheng BJ, He YQ, et al. Isolation and characterization of viruses related to the SARS coronavirus from animals in southern China. Science 2003;302:276-8.

- Ng EK, Hui DS, Chan KC, et al. Quantitative analysis and prognostic implication of SARS coronavirus in the plasma and serum of patients with severe acute respiratory syndrome. Clin Chem 2003;49:1976-80.

vitro antiviral activity of lopinavir/ritonavir against SARS CoV with lower rate of adverse outcome (defined as acute respiratory distress syndrome or death) of $2.4 \% \quad v \quad 28.8 \%$ $(\mathrm{p}<0.001)$ at day 21 after onset of symptoms in the treatment group than in the historical controls. ${ }^{61}$ The use of ribavirin in SARS in Toronto, based on a higher dosage for treating haemorrhagic fever virus, was associated with more significant toxicity, including haemolysis (in 76\%) and a decrease in haemoglobin of $20 \mathrm{~g} / \mathrm{l}$ (in 49\%), raised transaminases (in $40 \%$ ), and bradycardia (in $14 \%$ ). ${ }^{3}$

During phase 2 when there is radiological progression of pneumonia and/or hypoxaemia, intravenous high dose methylprednisolone $0.5 \mathrm{~g}$ daily has been given to prevent immunopathological lung injury, on the rationale that progression of the pulmonary disease may be mediated by the host inflammatory response. ${ }^{132}{ }^{62}$ The majority of a major cohort ( $89 \%$ of 138 patients) appeared to have a favourable response to the combination treatment with resolution of fever and lung opacities within two weeks. ${ }^{62}$ The use of high dose pulse methylprednisolone during clinical progression was associated with more favourable clinical improvement. ${ }^{62-64}$ In an uncontrolled study in Toronto, use of interferon alfacon-1 plus corticosteroids was associated with reduced disease-associated impaired oxygen saturation, more rapid resolution of radiographic lung opacities, and lower levels of creatine kinase. ${ }^{65}$ Nevertheless, any treatment regimen for SARS needs to tested with a randomised placebo controlled design. New antiviral agents and immunomodulating agents such as hyperimmune globulin and monoclonal antibody are also under investigation.

Non-invasive positive pressure ventilation has been used with success in SARS patients with respiratory failure. ${ }^{6366}$ However, non-invasive positive pressure ventilation should be carried out only if there is adequate protection for healthcare workers (for example, an isolation room with adequate air exchange) because of the potential risk of viral transmission via mask leakage and flow compensation causing dispersion of contaminated aerosol.

\section{PROGNOSIS/OUTCOME}

The poor prognostic factors associated with a poor outcome (intensive care unit admission or death) include advanced age, ${ }^{131} 3245$ chronic hepatitis B treated with lamivudine, ${ }^{32}$ high initial lactate dehydrogenase, ${ }^{45}$ high peak lactate dehydrogenase, ${ }^{1}$ high neutrophil count on presentation, ${ }^{145}$ diabetes mellitus or other co-morbid conditions, ${ }^{344}$ and low counts of CD4 and CD8 at presentation. ${ }^{38}$

\section{CONCLUSION}

With the major global outbreak of SARS in 2003 and its recent re-emergence in mainland China, ${ }^{67}$ further research in the development of rapid diagnostic tests and an effective treatment are urgently needed. The availability of the genome sequence of the SARS CoV will hopefully facilitate efforts to develop new and rapid diagnostic tests, antiviral agents, and effective vaccines in the long run. Animal species such as ferrets and domestic cats are susceptible to experimental infection by SARS CoV and may be useful as animal models to test antiviral drugs or vaccine candidates against SARS. ${ }^{68}$ Prevention of spread is most important for this highly infectious disease. Banning the sale of wild animals for human consumption, avoiding the use of nebulisers on general wards, isolation facilities, strict droplet precaution (hand hygiene, gown, gloves, N95 masks, eye protection) among healthcare workers managing SARS patients, contact tracing, and quarantine isolation for close contacts are all important measures.

\section{MULTIPLE CHOICE QUESTIONS (ANSWERS AFTER REFERENCES) \\ Q1. Children with SARS are more likely:}
(A) To require mechanical ventilation than adults
(B) To have higher fever than adults
(C) To have diarrhoea than adults
(D) To have milder signs and symptoms than adults
(E) To have neutropenia than adults

\section{Q2. Which ONE of the follow features is most commonly found in children with SARS? \\ (A) Myalgia \\ (B) Fever \\ (C) Hypothermia \\ (D) Increase in serum lactic dehydrogenase concentra- tion \\ (E) Increase in serum creatinine kinase concentration}

\section{Q3. Which of the following statements is false with regard to SARS?}

(A) Lymphopenia is a common laboratory feature.

(B) A high lactate dehydrogenase level on presentation is a poor prognostic marker

(C) Pleural effusion and cavitation are common radiographic features

(D) Cough and dyspnoea are not major complaints

(E) Respiratory failure is a common early complication

\section{Q4. Which of the following is not a poor prognostic factor for SARS?}
(A) Advanced age
(B) Diabetes mellitus
(C) Low lymphocyte count at presentation
(D) Fever $>40^{\circ} \mathrm{C}$
(E) High initial serum lactic dehydrogenase level 


\section{Q5. Which of the following is not a complication of SARS?}

(A) Disseminated intravascular coagulopathy

(B) Empyema

(C) Central nervous system involvement

(D) Cardiac dysfunction

(E) Pulmonary fibrosis

\section{Q6. Which of the following tests is the best in the early detection of SARS?}

(A) Nasopharyngeal aspirate for RT-PCR

(B) Stool for RT-PCR

(C) Urine for RT-PCR

(D) Serum RNA by real time RT-PCR

(E) Serology

\section{Authors' affiliations}

D S C Hui, M C H Chan, A K Wu, Department of Medicine and Therapeutics, Chinese University of Hong Kong, Prince of Wales Hospital, Shatin, New Territories, Hong Kong

P C Ng, Department of Paediatrics, Chinese University of Hong Kong,

Prince of Wales Hospital, Shatin, New Territories, Hong Kong

\section{REFERENCES}

1 Lee N, Hui DS, Wu A, et al. A major outbreak of severe acute respiratory syndrome in Hong Kong. N Engl J Med 2003;348:1986-94.

2 Hsu LY, Lee CC, Green JA, et al. Severe acute respiratory syndrome in Singapore: clinical features of index patient and initial contacts. Emerg Infect Dis 2003;9:713-17

3 Booth CM, Matukas LM, Tomlinson GA, et al. Clinical features and short-term outcomes of 144 patients with SARS in the greater Toronto area. JAMA 2003;289:2801-9

4 World Health Organisation. Summary of probable SARS cases with onset of illness from 1 November 2002 to 31 July 2003. Available at: http:// www.who.int/csr/sars/country/table2003_09_23/en/ (accessed 1 October 2003).

5 Peiris JS, Lai ST, Poon LL, et al. Coronavirus as a possible cause of severe acute respiratory syndrome. Lancet 2003;361:1319-25.

6 Kuiken T, Fouchier RA, Schutten M, et al. Newly discovered coronavirus as the primary cause of severe acute respiratory syndrome. Lancet 2003;362:263-70

7 Drosten C, Gunther S, Preiser W, et al. Identification of a novel coronavirus in patients with severe acute respiratory syndrome. N Engl J Med 2003;348:1967-76

8 Ksiazek TG, Erdman D, Goldsmith CS, et al. A novel coronavirus associated with severe acute respiratory syndrome. N Engl J Med 2003;348:1953-66.

9 Rota PA, Oberste MS, Monroe SS, et al. Characterization of a novel coronavirus associated with severe acute respiratory syndrome. Science 2003:300:1394-9.

10 Marra MA, Jones SJ, Astell CR, et al. The genome sequence of the SARSassociated coronavirus. Science 2003;300:1399-404.

11 Ruan YJ, Wei CL, Ee LA, et al. Comparative full-length genome sequence analysis of 14 SARS coronavirus isolates and common mutations associated with putative origins of infection. Lancet 2003;361:1779-85.

12 Zhao Z, Zhang F, Xu M, et al. Description and clinical treatment of an early outbreak of severe acute respiratory syndrome (SARS) in Guangzhou, PR China. J Med Micriobiol 2003;52:715-20.

13 Zhong NS, Zheng BJ, Li YM, et al. Epidemiology and cause of severe acute respiratory syndrome in Guangdong, People's Republic of China, in February 2003. Lancet 2003;362:1353-8.

14 Tsang KW, Ho PL, Ooi GC, et al. A cluster of cases of severe acute respiratory sundrome in Hong Kong. N Engl J Med 2003;348:1977-85.

15 Nicholls JM, Poon LL, Lee KC, et al. Lung pathology of fatal severe acute respiratory syndrome. Lancet 2003;361:1773-8.

16 Centers for Disease Control and Prevention. Severe acute respiratory syndrome. Fact sheet. Basic information about SARS, 8 May 2003. Available at: http://www.cdc.gov/ncidod/sars/factsheet.htm (accessed 12 May 2003)

17 Wong RS, Hui DS. Index patient and SARS outbreak in Hong Kong. Emerg Infect Dis 2004;10:339-41.

18 World Health Organisation. Acute respiratory syndrome in Hong Kong Special Administration of China/Vietnam. Available at: http://www.who.int/ csr/2003_03_12/en.

19 Kwan BC, Leung CB, Szeto CC, et al. Severe acute respiratory syndrome in a hemodialysis patient. Am J Kidney Dis 2003;42:1069-74.

20 Chim SS, Tsui SK, Chan KC, et al. Genomic characterization of the severe acute respiratory syndrome coronavirus of Amoy Gardens outbreak in Hong Kong. Lancet 2003;362:1807-8.
$21 \mathrm{Ng}$ SK. Possible role of an animal vector in the SARS outbreak at Amoy Gardens. Lancet 2003;362:570-2

22 World Health Organisation. Severe acute respiratory syndrome (SARS) multicountry outbreak. Available from: http://www.who.int/csr/2003_04_12/ en.

23 Centers for Disease Control and Prevention. Severe acute respiratory syndrome-Singapore, 2003. MMWR Morb Mortal Wkly Rep 2003;52:405-11.

24 Twu SJ, Chen TJ, Chen CJ, et al. Control measures for severe acute respiratory syndrome (SARS) in Taiwan. Emerg Infect Dis 2003;9:718-20.

25 Chiu RW, Chim SS, Lo YM. Molecular epidemiology of SARS-from Amoy Gardens to Taiwan. N Engl J Med 2003;349:1875-6.

26 World Health Organisation. Summary table of areas that experienced local transmission of SARS during the outbreak period from 1 November 2002 to 31 July 2003. Available at: http://www.who.int/csr/sars/areas/ areas2003_11_21/en.

27 Varia M, Wilson S, Sarwal S, et al. Investigation of a nosocomial outbreak of severe acute respiratory syndrome (SARS) in Toronto, Canada. CMAJ 2003;169:285-92

28 Centers for Disease Control and Prevention. Severe acute respiratory syndrome- Toronto, Canada, 2003. MMWR Morb Mortal Wkly Rep 2003;52:547-50.

29 World Health Organisation. Summary of probable SARS cases with onset of illness from 1 November to 31 July 2003. Available from: http:// www.who.int/csr/sars/country/table2003_09_23/en.

30 Guan Y, Zheng BJ, He YQ, et al. Isolation and characterization of viruses related to the SARS coronavirus from animals in southern China. Science 2003:302:276-8.

31 Donnelly CA, Ghani AV, Leung GM, et al. Epidemiological determinants of spread of causal agent of severe acute respiratory syndrome in Hong Kong. Lancet 2003;361:1761-6.

32 Peiris JS, Chu CM, Cheng VC, et al. Clinical progression and viral load in a community outbreak of coronavirus-associated SARS pneumonia: a prospective study. Lancet 2003;361:1767-72.

33 Leung WK, To KF, Chan PK, et al. Enteric involvement of severe acute respiratory syndrome-associated coronavirus infection. Gastroenterology 2003; 125:1011-17.

34 Hung EC, Chim SS, Chan PK, et al. Detection of SARS coronavirus RNA in the cerebrospinal fluid of a patient with severe acute respiratory syndrome. Clin Chem 49:2108-9.

35 Lau KK, Yu WC, Chu CM, et al. Possible central nervous system infection by SARS coronavirus. Emerg Infect Dis 2004; 10:342-4.

36 Wong KC, Leung KS, Hui M. Severe acute respiratory syndrome (SARS) in a geriatric patient with a hip fracture. A case report. J Bone Joint Surg Am 2003;85:1339-42.

37 Fisher DA, Lim TK, Lim YT, et al. Atypical presentations of SARS. Lancet 2003;361:1740

38 Wong RS, Wu A, To KF, et al. Haematological manifestations in patients with severe acute respiratory syndrome: retrospective analysis. BMJ 2003:326:1358-62.

39 Li SS, Cheng CW, Fu CL, et al. Left ventricular performance in patients with severe acute respiratory syndrome: a 30-day echocardiographic follow-up study. Circulation 2003;108:1798-803.

40 Wong WM, Ho JC, Hung IF, et al. Temporal patterns of hepatic dysfunction and disease severity in patients with SARS. JAMA 2003;290:2663-5.

41 Ooi CG, Khong PL, Lam B, et al. Severe acute respiratory syndrome: relationship between radiologic and clinical parameters. Radiology 2003;229:492-9.

42 Wong KT, Antonio GE, Hui DS, et al. severe acute respiratory syndrome: radiographic appearances and pattern of progression in 138 patients. Radiology 2003;228:401-6.

43 Wong KT, Antonio GE, Hui DS, et al. Thin section CT of severe acute respiratory syndrome: evaluation of 73 patients exposed to or with the disease. Radiology 2003;228:395-400.

44 Chan JW, Ng CK, Chan YH, et al. Short term outcome and risk factors for adverse clinical outcomes in adults with severe acute respiratory syndrome (SARS). Thorax 2003;58:686-9.

45 Tsui PT, Kwok ML, Yuen $\mathrm{H}$, et al. Severe acute respiratory syndrome: clinical outcome and prognostic correlates. Emerg Infect Dis 2003;9:1064-9.

46 Hon KL, Leung CW, Cheng WT, et al. Clinical presentations and outcome of severe acute respiratory syndrome in children. Lancet 2003;561:1701-3.

47 Chiu WK, Cheung PCH, Ng KL, et al. Severe acute respiratory syndrome in children: experience in a regional hospital in Hong Kong. Pediatr Crit Care Med 2003;4:279-83.

48 Bitnun A, Allen $\mathrm{U}$, Heurter $\mathrm{H}$, et al. Children hospitalized with severe acute respiratory syndrome-related illness in Toronto. Pediatrics 2003;112:e261-8.

49 Babyn PS, Chu WC, Tsou IY, et al. Severe acute respiratory syndrome (SARS) chest radiographic features in children. Pediatr Radiol 2004;34:47-58.

50 Shek CC, Ng PC, Fung GP, et al. Infants born to mothers with severe acute respiratory syndrome. Pediatrics 2003;112:e254-6.

51 Sit SC, Yau EKC, Lam YY, et al. A young infant with severe acute respiratory syndrome. Pediatrics 2003;112:e257-60.

52 World Health Organisation. Case definitions for surveillance of severe acute respiratory syndrome (SARS). Available at: http://www.who.int/csr/sars/ csedefinition/en/print.html (accessed 10 July 2003).

53 Rainer TH, Cameron PA, Smit D, et al. Evaluation of WHO criteria for identifying patients with severe acute respiratory syndrome out of hospital: prospective observational study. BMJ 2003;326:1354-8

54 World Health Organisation. Alert, verification and public health management of SARS in the post outbreak period. Available at: http://www.who.int/csr/ sars/postoutbreak/en/print.html (accessed August 17 2003). 
55 Centers for Disease Control and Prevention. Public health guidance for community-level preparedness and response to severe acute respiratory syndrome (SARS) version 2. Supplement B: SARS surveillance. Appendix B1: revised CSTE SARS surveillance case definition. Available at: http:// www.cdc.gov/ncidod/sars/guidance/B/pdf/b.pdf.

56 Poon LL, Chan KH, Wong OK, et al. Early diagnosis of SARS coronavirus infection by real time RT-PCR. J Clin Virol 2003;28:233-8.

$57 \mathrm{Ng}$ EK, Hui DS, Chan KC, et al. Quantitative analysis and prognostic implication of SARS coronavirus in the plasma and serum of patients with severe acute respiratory syndrome. Clin Chem 2003;49:1976-80.

$58 \mathrm{Ng}$ EK, $\mathrm{Ng} \mathrm{PC}$, Hon KL, et al. Serial analysis of the plasma concentration of SARS coronavirus RNA in pediatric patients with severe acute respiratory syndrome. Clin Chem 2003;49:2085-8.

59 Grant PR, Garson JA, Teddar RS, et al. Detection of SARS coronavirus in plasma by real-time RT-PCR. N Engl J Med 2003;349:2468-9.

60 Chan KS, Lai ST, Chu CM, et al. Treatment of severe acute respiratory syndrome with lopinavir/ritonavir: a multi-center retrospective matched cohort study. H K Med J 2003;9:399-406

61 Chu CM, Cheng VC, Hung IF, et al. Role of lopinavir/ritonavir in the treatment of SARS: initial virological and clinical findings. Thorax 2004;59:252-6.
62 Sung JJ, Wu A Joynt GM et al Severe acute respiratory syndrome: report of treatment and outcome after a major outbreak. Thorax 2004;59:414-20.

63 Nhong NS, Zeng GQ. Our strategies for fighting severe acute respiratory syndrome. Am J Respir Crit Care Med 2003;168:7-9.

64 Ho JC, Ooi GC, Mok TY, et al. High dose pulse versus non-pulse corticosteroid regimens in severe acute respiratory syndrome. Am J Respir Crit Care Med 2003; 168: 1449-56.

65 Loufty MR, Blatt LM, Siminovitch KA, et al. Interferon alfacon-1 plus corticosteroids in severe acute respiratory syndrome: a preliminary study. JAMA 2003;290:3222-8.

66 So LK, Lau AC, Yam LY, et al. Development of a standard treatment protocol for severe acute respiratory syndrome. Lancet 2003;361:1615-17.

67 World Health Organisation. New case of laboratory confirmed SARS in Guangdong, China-update 5. Available at: http://www.who.int/csr/don/ 2004 01 31/en.

68 Martina BE, Haagmans BL, Kuiken T, et al. SARS virus infection of cats and ferrets. Nature 2003;425:915.

ANSWERS

I. D; 2. B; 3. C; 4. D; 5. B; 6. D.

\section{IF ONLY I'D KNOWN}

\section{Ten things I wish I'd known when I was 25}

(1) Consultants regularly work longer hours than junior doctors.

(2) PFI [private finance initiative] economics don't make sense but the outcome was inevitable.

(3) Life is better outside major cities, but they are fun to visit.

(4) Do not apply for a consultant post in Tunbridge Wells if you decline to be involved in private practice.

(5) Medical journalism does not have the magical enriching effect of Harry Potter.

(6) Daughters are different from sons and more use on the whole.

(7) Medicine may be a doctor's principal hobby, but it should not be the only one.

(8) The NHS has higher standards than private practice despite the negative publicity, and tackles all the most difficult problems.

(9) Conscientious teachers put in just as many hours as doctors.

(10) Health service managers have a much shorter professional life than consultants, who are able to take a longer view.

M Bateson

Bishop Auckland General Hospital, Bishop Auckland, UK batesonm@smtp.sdhc-tr.northy.nhs.uk 